\title{
Baade-Wesselink distances and the effect of metallicity in classical cepheids
}

\author{
M. A. T. Groenewegen ${ }^{1,2}$ \\ 1 Koninklijke Sterrenwacht van België, Ringlaan 3, 1180 Brussels, Belgium \\ e-mail: marting@oma.be \\ 2 Instituut voor Sterrenkunde, Celestijnenlaan 200 D, 3001 Leuven, Belgium
}

Received 27 March 2008 / Accepted 3 June 2008

\section{ABSTRACT}

\begin{abstract}
Context. The metallicity dependence of the Cepheid $P L$-relation is of importance in establishing the extra-galactic distance scale. Aims. The aim of this paper is to investigate the metallicity dependence of the $P L$-relation in $V$ and $K$ based on a sample of 68 Galactic Cepheids with individual Baade-Wesselink distances (some of the stars also have an HST-based parallax) and individually determined metallicities from high-resolution spectroscopy.

Methods. Literature values of the $V$-band, $K$-band and radial velocity data have been collected for a sample of 68 classical cepheids that have their metallicity determined in the literature from high-resolution spectroscopy. Based on a $(V-K)$ surface-brightness relation and a projection factor derived in a previous paper, distances have been derived from a Baade-Wesselink analysis. $P L$ - and $P L Z$-relations in $V$ and $K$ are derived.

Results. The effect of the adopted dependence of the projection factor on period is investigated. The change from a constant $p$-factor to one recently suggested in the literature with a mild dependence on $\log P$ results in a less steep slope by 0.1 unit, which is about the 1 -sigma error bar in the slope itself. The observed slope in the $P L$-relation in $V$ in the LMC agrees with both hypotheses. In $K$ the difference between the Galactic and LMC slope is larger and would favour a mild period dependence of the $p$-factor. The dependence on metallicity in $V$ and $K$ is found to be marginal, and independent of the choice of $p$-factor on period. This result is severely limited by the small range in metallicity covered by the Galactic Cepheids.
\end{abstract}

Key words. stars: distances - stars: variables: cepheids - cosmology: distance scale - stars: oscillations including pulsations

\section{Introduction}

Obtaining accurate distances to stars is a non-trivial matter. Cepheids are considered an important standard candle as they are bright and are thus the link between the distance scale in the nearby universe and that further out via those galaxies that contain both Cepheids and SNIa.

Distances to local cepheids may be obtained via mainsequence fitting for those Cepheids in clusters (e.g. Feast 1999) or via determination of the parallax. Until recently only Polaris had an accurate parallax determination via Hipparcos. Benedict et al. (2007) recently published absolute trigonometric parallaxes for nine Galactic Cepheids using the Fine Guidance Sensor on board the Hubble Space Telescope, and revised, more accurate, Hipparcos parallaxes have also become available (van Leeuwen et al. 2007; van Leeuwen 2007).

In addition, distances to Cepheids can be obtained from the Baade-Wesselink method. This method relies on the availability of surface-brightness $(\mathrm{SB})$ relations to link variations in colour to variations in angular diameters and understanding of the projection $(p-)$ factor that links radial velocity to pulsational velocity variations.

The SB relations can be obtained using Cepheids that have accurate interferometrically determined angular diameters over the pulsation phase and accurate multi-wavelength light curves. This has allowed Kervella et al. (2004) and Groenewegen (2007, hereafter G07) to derive such relations, in particular using the $(V-K)$ colour, which gives the highest precision in the derived angular diameter.
The $p$-factor can also be obtained from Cepheids with interferometrically determined angular diameters over the pulsation phase when in addition accurate RV curves are available (see e.g. Mérand et al. 2005). G07 assembled all currently available data to find that a constant $p$-factor represents the best fit to the available data. A strong dependence on period, like $p \sim-0.15 \log P$ as proposed by Gieren et al. (2005), could be ruled out. A moderate dependence of $p \sim-0.03 \log P$ as used in the Gieren et al. papers (1993, 1997, 1998; Storm et al. 2004; Barnes et al. 2003), or $p \sim-0.064 \log P$ as advocated by Nardetto et al. (2007) are also consistent with the available data.

Another aspect of the Cepheid $P L$-relation that is still a matter of debate is the metallicity dependence of the Cepheid $P L$-relation, and its dependence on wavelength. Observations seem to consistently indicate that metal-rich Cepheids are brighter, and various estimates have been given in the literature, $-0.88 \pm 0.16 \mathrm{mag} / \mathrm{dex}$ (BRI bands, Gould 1994), $-0.44_{-0.2}^{+0.1} \mathrm{mag} / \mathrm{dex}$ ( $V R$ bands, Sasselov et al. 1997), $-0.24 \pm$ $0.16 \mathrm{mag} / \mathrm{dex}$ ( $V I$ bands, Kochanek 1997), $-0.14 \pm 0.14 \mathrm{mag} / \mathrm{dex}$ ( $V I$ bands, Kennicutt et al. 1998), $-0.21 \pm 0.19$ in $V,-0.29 \pm 0.19$ in $W,-0.23 \pm 0.19$ in $I,-0.21 \pm 0.19 \mathrm{mag} / \mathrm{dex}$ in $K$ (Storm et al. 2004), and $-0.29 \pm 0.10 \mathrm{mag} / \mathrm{dex}$ (BVI bands, Macri et al. 2006).

The potential caveat is that no individual abundance determinations of individual Cepheids are being used in these studies but rather abundances of nearby HII regions, or even a mean abundance of the entire galaxy.

Groenewegen et al. (2004) tried to attack the problem by using Cepheids with individually determined metallicities, 37 in 
our Galaxy, 10 in the LMC and 6 in the SMC. Considering the Galactic sample, a metallicity effect was found in the zero point in the VIWK PL-relation of $-0.6 \pm 0.4$ or $-0.8 \pm 0.3 \mathrm{mag} / \mathrm{dex}$, depending on the in- or exclusion of one object. The distances to the galactic Cepheids mostly came from BW-distances available at that time from Fouqué et al. (2003).

In the last few years significant progress has been made in several areas. On the one hand more Cepheids have interferometrically determined angular diameters. Combined with improved theoretical studies (Nardetto et al. 2007, and references therein) this has led to an improved understanding of the $p$-factor and SB relations, as was studied in G07. On the other hand, more basic photometric light curves and radial velocity data have become available and new studies have presented metallicity determinations for significant samples (see references below).

In this paper, Baade-Wesselink distances to Cepheids with metallicity determinations are revisited, using the $p$-factor and the SB $(V-K)$ colour relation derived in G07, updated as described in Sect. 3. Section 2 describes the selection of the photometric and radial velocity data. Section 3 outlines how the data are modelled. Section 4 describes how the binary Cepheids are treated, and new and updated orbital elements are presented. Section 5 describes the results. Section 6 presents conclusions.

\section{The sample}

In a first step Cepheids with recent (post-2000) accurate metallicity determinations were selected (from Andrievsky et al. 2002a,b,c, 2003-2005; Luck et al. 2003, 2006; Luck \& Andrievsky 2004; Kovtyukh et al. 2005a,b; Yong et al. 2006; Lemasle et al. 2007; Mottini et al. 2008), and for those, the McMaster Cepheid Photometry and Radial Velocity Data Archive $^{1}$ was checked for the availability of $V, K$ and radial velocity (RV) data. For the stars selected, the literature was searched for additional data.

Table 1 lists the 68 stars selected with the source of data indicated. The last column indicates if particular datasets and/or ranges in Julian date (JD) were not used in the fitting described in the next section to derive the distance. This is typically because these datasets show very large residuals, or the stars show a noticeable change or shift in period, and the range in JD had to be restricted, typically centred on the available $K$-magnitude data. For the determination of the orbital parameters of the Cepheids in binaries (Sect. 4), all available RV data were used.

For four Cepheids new high-resolution spectra were obtained in order to derive the radial velocity ${ }^{2}$. Observations took place in July and August 2007 using the Coralie spectrograph at the $1.2 \mathrm{~m}$ Euler telescope located at the La Silla observatory, Chile. This is a fibre-fed echelle spectrograph ( $2^{\prime \prime}$ fibres with one on the object and one on the sky), which covers the 3880 to $6810 \AA$ region in 68 orders with a spectral resolution of about 50 000. Exposure times are between 3 and 10 min. During each night, several exposures with a tungsten lamp were taken to measure the relative pixel sensitivity variation of the CCD. A Thorium-Argon lamp was observed at the beginning and end of the night for wavelength calibration. The Heliocentric velocities are obtained as part of the standard pipeline reduction whereby the spectrum is cross-correlated against a mask, in this case that of a G2 star.

\footnotetext{
1 http://crocus.physics.mcmaster.ca/Cepheid/

2 Two stars are no longer considered in the Baade-Wesselink analyses below: BQ Ser and V367 Sct which are classified as double-mode Cepheids.
}

The formal precision on the RVs is of the order $5-15 \mathrm{~m} / \mathrm{s}$. The RVs are listed in Table 2.

Like in G07, in a first step to attempt to homogenise the datasets, the light curves are read in the program Period04 (Lenz $\&$ Breger 2005), which allows for an easy visualisation of different datasets. Period04 is used to calculate the period, and phase the data to see if there are clear outliers in the data (which are flagged and excluded) or clear off-sets between datasets. Some datasets were in the end not considered because of very large error bars. The rms in the fit was determined for each dataset separately and this was used as the typical error bar (unless the rms was consistent with the original error bars quoted, in which case these were retained).

$K$-band data on the CIT/CTIO system were transformed to the SAAO-Carter system using the formula in Carter (1990), and Johnson and SAAO-Glass IR-photometry was transformed to the SAAO-Carter system according to Glass (1985).

Table 3 lists the adopted metallicity value. They were taken preferentially from Luck \& Andrievsky (2004), Kovtyukh et al. (2005), and Andrievsky et al. (2005) who studied the fundamental parameters, including abundances, at several phases. Alternatively, values were taken from other papers by Andrievsky/Luck/Kovtyukh, and averaged when multiple determinations were available. Thirdly, iron abundances from Fry \& Carney (1997) and Yong et al. (2006) were transformed to the "Andrievsky system" following Laney \& Caldwell (2007) and Luck et al. (2006). Based on the 12 stars in common between Andrievsky et al. (2003) and Mottini et al. (2008) an offset AND - MOT $=+0.06 \pm 0.11$ was determined, and based on 6 stars in common with Lemasle et al. (2007) an offset AND LEM $=+0.09 \pm 0.07$. These offsets were applied to the Mottini et al. and Lemasle et al. iron abundances to bring them to the "Andrievsky system".

\section{The model}

The model is outlined in G07 but will be briefly repeated here. The $V$ -,$K$ - and RV data with error bars are fitted with a function of the form:

$F(t)=F_{0}+\sum_{i=1}^{i=N}\left(A_{i} \sin \left(2 \pi t \mathrm{e}^{i f}\right)+B_{i} \cos \left(2 \pi t \mathrm{e}^{i f}\right)\right)$

where $P=\mathrm{e}^{-f}$ is the period (in days). Typically, $P$ is determined from the fit to the available optical photometry as this dataset is usually most extensive. The period is then fixed when fitting Eq. (1) to the $K$-band and RV data.

The determination of the parameters is done using the MRQMIN routine (using the Levenberg-Marquardt method) from Press et al. (1992) written in Fortran77, which minimises

$\chi^{2}=\sum_{i=1}^{i=n}\left(F_{i}-F\left(t_{i}\right)\right)^{2} /\left(\sigma_{F_{i}}\right)^{2}$

with $F_{i}$ the measurement at time $t_{i}$ which has an error bar $\sigma_{F_{i}}$. Also the reduced $\chi^{2}$ is defined:

$\chi_{\mathrm{r}}^{2}=\frac{\chi^{2}}{(n-p)}$

and the quantity

$\mathrm{BIC}=\chi^{2}+(p+1) \ln (n)$, 
Table 1. Sources of $V$-, $K$-band and RV data.

\begin{tabular}{|c|c|c|c|c|}
\hline Name & $V$ & $K$ & $R V$ & Data not considered $^{1}$ \\
\hline AQ Pup & $1,2,3,4,5,84$ & $6,7,8$ & $1,10,11,44$ & $3,-,-$ JD $<34000$, JD $>49700$ \\
\hline BB Sgr & $2,3,16,63$ & 6,24 & $20,33,39,44$ &,,-- 44 \\
\hline beta Dor & $3,11,16$ & 6,18 & $11,19,20,21,22$ &,,-- 20 \\
\hline BF Oph & $2,3,16,23,63$ & $6,18,24$ & $10,20,39,44,76$ & $\mathrm{JD}<36000$ \\
\hline BG Lac & $2,3,12,13$ & 12 & $14,15,44$ & \\
\hline BN Pup & $1,3,5,16$ & 6,7 & 1,17 & \\
\hline CD Cyg & $2,3,64$ & 24 & 33,44 & \\
\hline CV Mon & $2,3,13,16,23$ & 6,24 & $25,26,44$ & \\
\hline delta Cep & $2,3,11,12,13,27$ & 12,98 & $4,9,14,25,28,29$ &,,$-- 9 \mathrm{JD}<43500$ \\
\hline DT Cyg & $2,3,4,27,30,31$ & 24,32 & $4,9,28,29,33,34$ & $-, 32,9+28 \mathrm{JD}<34000$ \\
\hline eta Aql & $2,3,12,13,16,27,35$ & 12,24 & $9,14,20,25,28,29,36,37$ &,,-- 9 \\
\hline FF Aql & $2,3,11,16,27,30,31$ & $18,24,32$ & $9,20,28,29,33,38$ & $3,32,9 \mathrm{JD}<44000$ \\
\hline FM Aql & $2,3,12,13,16,23$ & 12,24 & $14,33,39,44$ & $\mathrm{JD}<30000$ \\
\hline FN Aql & $2,3,12,13,16,23$ & 12,24 & $14,33,39$ &,,-- 39 \\
\hline KN Cen & $1,3,5,11,16,40,84$ & 6 & $1,17,41$ & $-,-, 41 \mathrm{JD}<40000, \mathrm{JD}>47000$ \\
\hline KQ Sco & $1,3,11$ & 6 & 1,42 & \\
\hline $1 \mathrm{Car}$ & $3,5,11,16,35$ & 6 & $11,19,20,23,43$ & $5,-, 23 \mathrm{JD}<46500$ \\
\hline LS Pup & $1,3,11$ & 6 & 1 & \\
\hline RS Pup & $2,3,5,11,16,84$ & $6,8,24$ & $11,19,25,44$ & $-,-, 44 \mathrm{JD}<42000, \mathrm{JD}>50000$ \\
\hline RT Aur & $2,12,27,31,45$ & 12,32 & $9,28,29,33,37,46$ &,,-- 9 \\
\hline RU Sct & $2,3,16$ & 6 & $26,33,105$ & \\
\hline RY Sco & $1,2,3,5,11,16$ & 6 & $1,20,44$ & \\
\hline RY Vel & $1,3,5,11,16$ & 6 & $1,11,17$ & $\mathrm{JD}<44000, \mathrm{JD}>50000$ \\
\hline RZ Vel & $1,3,5,11,16$ & 6 & $1,11,20,47$ & \\
\hline S Mus & $3,11,23,40$ & 6,24 & $10,11,20,22,41,47,48,49,50$ &,,$-- 41 \mathrm{JD}<30000$ \\
\hline S Nor & $3,11,23,40$ & 6,24 & $4,10,20,41,47,49,51,52,53$ &,,$-- 41 \mathrm{JD}<30000$ \\
\hline S Sge & $2,3,11,12,13,23,27,30,40$ & $12,24,32$ & $9,14,28,29,33,37,54,55,56$ & $-, 32,54$ \\
\hline SS Sct & $2,3,16,23,63$ & 24 & $39,42,44,63$ & $\mathrm{JD}<30000$ \\
\hline SU Cas & $2,3,9,27,31$ & 12,32 & $4,9,25,28,29,33,57,58,59,60,61,62$ & $-, 32,9 \mathrm{JD}<43000$ \\
\hline SU Cyg & $2,3,27,30,31$ & 24 & $9,28,33,106,107,108,109$ &,,$-- 9 \mathrm{JD}<35000$ \\
\hline S Vul & 3,30 & 6 & 33,110 & $\mathrm{JD}<45400$ \\
\hline SV Vul & $2,3,4,9,27,64$ & $6,12,24$ & $4,9,14,15,25,28,29,33,39,65$ & $\mathrm{JD}<45500, \mathrm{JD}>48600$ \\
\hline SW Vel & $1,3,5,11,16,84$ & 6 & 1,11 & \\
\hline SX Vel & $3,23,40$ & 6 & 10 & \\
\hline SZ Aql & $2,3,11,12,16,23,64$ & $6,12,24$ & $11,14,23$ & \\
\hline SZ Tau & $2,3,4,12,27,31$ & $6,12,32$ & $4,9,28,29,33$ & $-, 32,9 \mathrm{JD}<43000, \mathrm{JD}>48000$ \\
\hline T Mon & $1,2,3,11,16,35,64$ & $6,24,32$ & $4,9,25,29,33,37,48,65,66,67,68,69,70,71,72,73$ & $-, 32,70 \mathrm{JD}<44500$ \\
\hline TT Aql & $2,3,11,12,16,27,64,74$ & 12,24 & $9,11,14,15,22,28,33,37,39,74$ &,,$-- 74 \mathrm{JD}<44000$ \\
\hline T Vel & $3,16,75$ & 6,18 & $10,20,75$ & $-, 18,-$ \\
\hline T Vul & $2,3,12,27,31$ & $12,24,32,98$ & $4,9,14,28,29$ & $-, 32,9 \mathrm{JD}<43600$ \\
\hline U Car & $1,3,5,11,16,35$ & 6,24 & $1,11,20$ & \\
\hline U Nor & $1,5,11,16$ & 6 & $1,11,17,41$ & \\
\hline U Sgr & $2,3,4,16,23,35,63$ & $6,24,32$ & $4,9,10,11,20,25,28,33,39,47,51,76,77,78$ & $-, 32,9 \mathrm{JD}<37000$ \\
\hline UU Mus & $1,5,11,40,84$ & 6 & 1,11 & \\
\hline U Vul & $2,3,12,13,23,27$ & 12 & $4,14,33,37,44,79,80$ &,,-- 44 \\
\hline V340 Nor & $3,4,82$ & 6 & 4,26 & \\
\hline V350 Sgr & $2,3,23,63,99$ & 18,24 & $20,22,33,44,76,83,39$ & $-, 24,-\mathrm{JD}<42000$ \\
\hline V496 Aql & $2,3,16,63$ & 24 & $9,10,20,33,76$ & \\
\hline V Car & $3,16,23$ & 6 & $10.20,47$ & \\
\hline V Cen & $3,11,16,23,63$ & 6,24 & $10,11,20,47,76$ & $-, 24,-\mathrm{JD}<42000$ \\
\hline VW Cen & $1,3,16,84$ & 6 & 1,41 &,,-- 41 \\
\hline VY Car & $1,3,5,11,16,99,100$ & 6,24 & $1,11,20,41$ & $\mathrm{JD}<42000$ \\
\hline VZ Cyg & $2,3,12,31$ & 12,24 & $4,14,33,39,44$ & $\mathrm{JD}<40000$ \\
\hline VZ Pup & $1,3,5,11,16$ & $6,7,8$ & 1,11 & \\
\hline W Sgr & $2,3,40,85$ & $24,32,86$ & $4,12,20,22,28,47,48,85,87,88,89$ & \\
\hline WZ Car & $1,3,5,11,16$ & 6 & 1,11 & \\
\hline WZ Sgr & $1,2,3,5,11,40$ & 6 & $1,11,33,39$ & $\mathrm{JD}<40000$ \\
\hline X Cyg & $2,3,27$ & $12,24,32,98$ & $4,14,25,29,33$ & \\
\hline X Lac & $2,3,12,13$ & 12 & $4,14,33,44$ &,,-- 44 \\
\hline X Pup & $2,3,5,11,16,23$ & 6 & $11,23,44$ & $5,-,-$ \\
\hline X Sgr & $2,3,11,16,35,99$ & $24,32,104$ & $9,10,11,20,28,47,52,101,102,103$ &,,$-- 9 \mathrm{JD}<35000$ \\
\hline XX Cen & $3,11,40,74$ & 6,24 & $10,11,20,41,52,74$ & $-, 24,74$ \\
\hline Y Lac & $2,3,12,31,90$ & 12 & $14,15,33,39,44$ & $\mathrm{JD}<26500$ \\
\hline Y Oph & $1,2,3,16$ & 6,24 & $1,9,19,20,28,33,34,91,92$ &,,$-- 9 \mathrm{JD}<40000$ \\
\hline
\end{tabular}


Table 1. continued.

\begin{tabular}{rrrrl}
\hline \hline Name & $V$ & $K$ & $R V$ & Data not considered $^{1}$ \\
\hline Y Sgr & $2,3,11,16,23,35$ & 24,32 & $9,11,19,20,28,46,49,93,94$ &,,-- 9 JD $<28500$ \\
YZ Sgr & $2,3,23,40$ & 24 & 20,44 & \\
Z Lac & $2,3,12,64$ & 12 & $14,33,39,44,80,95,96,97$ & JD $<40500$ \\
zeta Gem & $2,3,4,27,35,64$ & 32,104 & $4,9,19,28,29,33,37$ &,,-- 9 \\
\hline
\end{tabular}

References:

1 = Coulson \& Caldwell (1985a); 2 = Moffett \& Barnes (1984); 3 = Berdnikov et al. (2000), a datafile named "cepheids-16-03-2006" was retrieved from the ftp address listed in that paper; $4=$ Bersier et al. (1994); $5=$ Madore (1975); $6=$ Laney \& Stobie (1992); 7 = Schechter et al. (1992); $8=$ Welch (1985); 9 = Barnes et al. (1987), points with uncertainty flag “:” were removed; $10=$ Stibbs (1955); $11=$ Bersier (2002), data points with weight 0 and 1 in the Geneva photometry were removed; $12=$ Barnes et al. (1997); $13=$ Szabados $(1980) ; 14=$ Barnes et al. $(2005) ; 15=$ Imbert (1999); 16 = Pel (1976); 17 = Pont et al. (1994); 18 = Lloyd Evans (1980a); $19=$ Nardetto et al. (2006); $20=$ Lloyd Evans (1980b); 21 = Taylor \& Booth (1998); 22 = Petterson et al. (2005); 23 = Caldwell et al. (2001); 24 = Welch et al. (1984); $25=$ Storm et al. (2004); $26=$ Metzger et al. (1992); $27=$ Kiss (1998); $28=$ Wilson et al. (1989); $29=$ Kiss (2000); $30=$ Szabados (1991); $31=$ Szabados (1977); $32=$ Wisniewski \& Johnson (1968); 33 = Gorynya et al. (1998, VizieR On-line Data Catalog: III/229); $34=$ Sanford (1935); $35=$ Shobbrook (1992); $36=$ Jacobsen \& Wallerstein (1981); 37 = Evans (1976); 38 = Evans et al. (1990); 39 = Joy (1937); 40 = Walraven et al. (1964); $41=$ Grayzeck (1978); 42= This paper, Table 1; 43 = Taylor et al. (1997); $44=$ Barnes et al. (1988), points with uncertainty flag ":" were removed; $45=$ Turner et al. (2007); 46= Duncan (1908); 47 = Lloyd Evans (1968); $48=$ Petterson et al. (2004); $49=$ Campbell \& Moore (1928); 50 = Böhm-Vitense et al. (1990); 51 = Mermilliod et al. (1987); 52 = Feast (1967); $53=$ Breger (1970); $54=$ Evans et al. (1993); $55=$ Herbig \& Moore (1952); $56=$ Breitfellner \& Gillet (1993); 57 = Adams \& Shapley (1918); $58=$ Abt (1959); 59= Niva \& Schmidt (1979); 60 = Gieren (1976); $61=$ Häupl (1988); 62= Beavers \& Eitter (1986); $63=$ Gieren (1981b); $64=$ Szabados (1981); $65=$ Sanford (1956); $66=$ Evans et al. (1999); $67=$ Frost $(1906) ; 68=$ Sanford (1927); $69=$ Wallerstein (1972); $70=$ Coulson (1983); $71=$ Evans \& Lyons (1994); $72=$ Gieren (1989); $73=$ Harper (1934); $74=$ Coulson et al. (1985); $75=$ Gieren (1985); $76=$ Gieren (1981a); $77=$ Jacobsen (1970); $78=$ Breger (1967); 79= Sanford (1951); 80= Imbert (1996); 81 = Coulson \& Caldwell (1985b); 82 = Eggen (1983); 83 = Evans \& Sugars (1997); 84 = Hipparcos Epoch Photometry; $85=$ Babel et al. (1989); 86= Kimeswenger et al. (2004); 87 = Albrow \& Cottrell (1996); 88 = Jacobsen et al. (1984); $89=$ Jacobsen (1974); $90=$ Henden (1980); $91=$ Evans \& Lyons (1992); 92 = Abt \& Levy (1978); 93 = Duncan (1922); 94 = ten Bruggencate (1930); 95 = Evans \& Welch (1993); $96=$ Gieren (1989); $97=$ Sugars \& Evans (1996); 98 = Fernley et al. (1989); $99=$ Dean (1977); $100=$ Dean (1981); $101=$ Duncan (1932); 102= Slipher $(1904) ; 103=$ Moore (1909); 104 = Feast et al. (2008); 105 = Metzger et al. (1993); 106 = Evans (1988); 107 = Maddrill (1906); 108 = Abt (1973); 109= Imbert (1985); $110=$ Joy (1952).

Note: $1=$ The number indicates the dataset not used in, respectively, $V, K, \mathrm{RV}$.

where $p=2 N+2$ is the number of free parameters $(p=2 N+1$ when fitting the RV and $K$ light curve). As the number $N$ of harmonics to be fitted to the data is a priori not known, one could obtain ever better fits (lower $\chi^{2}$ ) by increasing $N$. The Bayesian information criterion (Schwarz 1978) is a formalism that penalises this, and $N$ (for the $V, K$ and RV curve independently) is chosen such that BIC reaches a minimum. The number of harmonics used varies between 3 and 10 in the optical, 1 and 5 in the NIR, and 2 and 9 for the RV curves.

Given the analytical form of Eq. (1), the radial velocity curve can be integrated exactly to obtain the variation in radius as a function of time (phase):

$\Delta R(t, \delta \theta)=-p \int_{t_{0}}^{t+P \delta \theta}\left(v_{\mathrm{R}}-\gamma\right) \mathrm{d} t$,

where $\gamma$ is the systemic velocity, $v_{\mathrm{R}}$ the radial velocity, $p$ the projection factor and $\delta \theta$ allows for a phase shift between the RV curve and the angular diameter variations determined via the SB relation.

Then, the equation

$\theta(t)=9.3038 \operatorname{mas}\left(\frac{R_{0}+\Delta R(t, \delta \theta)}{d}\right)$

is fitted with $\theta$ the angular diameter in mas, $R_{0}$ the stars radius in solar radii and $d$ the distance in pc. The fitting is done using the linear bi-sector (using the code SIXLIN from Isobe et al. 1990) as used and preferred by e.g. Storm et al. (2004), Barnes et al. (2005) and Gieren et al. (2005). In some cases a phase range around $0.85-0.95$ was excluded from the fit.

In G07 the $p$-factor was determined chiefly using six Cepheids with interferometrically measured angular diameter variations and HST based accurate parallaxes
(Benedict et al. 2007). It was concluded that, formally, there was no need for a period-dependent $p$-factor, and $p=1.27$ \pm 0.05 fitted the available data. On the other hand, a moderate dependence of $p \sim-0.03 \log P$ as used in the Gieren et al. papers (1993, 1997, 1998; Storm et al. 2004; Barnes et al. 2003), or $p \sim-0.064 \log P$ as advocated by Nardetto et al. (2007) are also consistent with the available data.

The Nardetto et al. (2007) theoretical investigation suggest that there is a difference between the $p$-factor to be used with wide-band interferometry (like in G07) and with RV data (when applying the SB technique as in the present study). For $\delta$ Cep this difference is of the order of 0.06 (Nardetto et al. 2004, 2007).

In the present paper, two $p$-factors will be considered: $p=$ 1.33 (the value found in G07 corrected by 0.06 ), and $p=1.376-$ $0.064 \log P$ from Nardetto et al. (2007). The derived distances depend linearly on the adopted value of $p$. 1999):

An SB relation can be defined as follows (see van Belle

$\theta_{o}=\theta \times 10^{\left(m_{1} / 5\right)}$,

where $\theta$ is the LD angular diameter (in mas), and $m_{1}$ a dereddened magnitude (for example, $V_{0}$ ). The logarithm of this quantity (the zero magnitude angular diameter) is plotted against a de-reddened colour (for example, $\left.(V-K)_{0}\right)$,

$\log \theta_{0}=a \times\left(m_{2}-m_{3}\right)+b$.

G07 analysed the available datasets of Cepheids with interferometrically determined angular diameters over the light curve and derived a SB relation. In a recent paper, Feast et al. (2008) presented new NIR data for zeta Gem and X Sgr. Taking this new data into account, Eq. (9) in G07 becomes

$\log \theta_{0}=(0.2724 \pm 0.0045)(V-K)_{0}+(0.5283 \pm 0.0091)$ 
Table 2. New radial velocity data.

\begin{tabular}{|c|c|c|c|}
\hline \multicolumn{2}{|c|}{$R V\left(\mathrm{~km} \mathrm{~s}^{-1}\right)$} & \multicolumn{2}{|c|}{$R V\left(\mathrm{~km} \mathrm{~s}^{-1}\right)$} \\
\hline \multicolumn{2}{|c|}{ KQ Sco } & \multicolumn{2}{|c|}{ V367 Sct } \\
\hline 2454302.678 & -7.57 & 2454341.605 & -22.61 \\
\hline 2454303.670 & -10.77 & 2454341.605 & 26.97 \\
\hline 2454304.527 & -26.89 & 2454302.737 & -19.57 \\
\hline 2454307.627 & -45.12 & 2454303.709 & -19.15 \\
\hline 2454308.672 & -45.51 & 2454305.682 & 0.77 \\
\hline 2454309.722 & -53.38 & 2454306.754 & 4.38 \\
\hline 2454310.570 & -49.50 & 2454307.780 & -16.31 \\
\hline 2454311.711 & -48.46 & 2454308.741 & -16.90 \\
\hline 2454312.605 & -50.97 & 2454310.747 & -5.25 \\
\hline 2454332.497 & -12.06 & 2454311.733 & -7.84 \\
\hline 2454336.489 & -47.39 & 2454312.762 & -9.21 \\
\hline 2454339.524 & -42.66 & 2454332.588 & 5.21 \\
\hline \multirow[t]{2}{*}{2454341.537} & -46.58 & 2454334.567 & -20.65 \\
\hline & & 2454334.581 & -20.30 \\
\hline \multicolumn{2}{|c|}{ SS Sct } & 2454339.626 & -13.20 \\
\hline 2454302.580 & 7.55 & 2454341.580 & -9.54 \\
\hline 2454303.739 & -24.37 & \multirow{2}{*}{\multicolumn{2}{|c|}{ BQ Ser }} \\
\hline 2454304.754 & -11.28 & & \\
\hline 2454305.772 & 3.70 & 2454302.601 & -18.97 \\
\hline 2454306.711 & 1.72 & 2454303.731 & -11.41 \\
\hline 2454307.691 & -23.22 & 2454304.724 & -15.08 \\
\hline 2454308.624 & -8.05 & 2454305.738 & -11.01 \\
\hline 2454309.616 & 5.48 & 2454306.731 & -1.57 \\
\hline 2454310.785 & -17.19 & 2454307.747 & -27.11 \\
\hline 2454311.656 & -18.74 & 2454308.717 & -17.00 \\
\hline 2454312.806 & -0.36 & 2454309.737 & -4.14 \\
\hline 2454331.699 & 6.05 & 2454310.705 & -2.98 \\
\hline 2454332.556 & -4.74 & 2454311.753 & -22.50 \\
\hline 2454334.613 & -3.38 & 2454312.718 & -11.03 \\
\hline 2454335.533 & 7.01 & 2454331.653 & 1.03 \\
\hline 2454336.671 & -23.15 & 2454332.517 & -23.78 \\
\hline 2454339.644 & 5.00 & 2454334.536 & -10.36 \\
\hline \multirow{3}{*}{2454341.637} & -8.36 & 2454335.515 & -13.03 \\
\hline & & 2454339.557 & -6.35 \\
\hline & & 2454341.559 & -25.73 \\
\hline
\end{tabular}

which is the form of the the SB relation used in this paper.

Colour excess values $E(B-V)$ used to de-redden the $V$ and $K$ magnitudes have been taken from the compilation by Fouqué et al. (2007, hereafter F07). Reddening ratios of $R_{V}=3.3$ and $A_{K} / A_{V}=0.0909$ have been adopted, as in G07. For the stars already studied in G07 (beta Dor, 1 Car, zeta Gem, W Sgr, Y Sgr, del Cep, FF Aql, T Vul, RT Aur), for consistency, the $E(B-V)$ values from G07 have been taken, which are based on the $A_{V}$ values in Benedict et al. (2007) and the reddening ratio of 3.3. The resulting $E(B-V)$ values do not differ significantly from the values listed in F07.

\section{Binary cepheids}

A number of stars in the sample are known or suspected spectroscopic binaries. In order to apply the SB technique outlined in the previous section, the RV data have to be corrected for the binary motion.

The database on Cepheids in binary systems ${ }^{3}$ (Szabados 2003) is most useful as it lists published orbital parameters and references. For some stars, more RV data have become available than was used in the last paper that published an orbit and so it was decided to rederive the 5 orbital parameters (in the case of the eccentricity, $\ln e$ was the parameter actually fitted).

${ }^{3}$ http://www.konkoly.hu/CEP/intro.html
Table 3. Adopted $[\mathrm{Fe} / \mathrm{H}]$ values for the Cepheids in the sample.

\begin{tabular}{|c|c|c|c|}
\hline Name & {$[\mathrm{Fe} / \mathrm{H}]$} & Name & {$[\mathrm{Fe} / \mathrm{H}]$} \\
\hline AQ Pup & $-0.14(5)$ & SZ Aql & $+0.17 \pm 0.04(2)$ \\
\hline BB Sgr & $+0.08(6)$ & SZ Tau & +0.08 (4) \\
\hline beta Dor & $-0.01(4)$ & T Mon & $+0.15 \pm 0.03$ \\
\hline BF Oph & $+0.00(6)$ & TT Aql & $+0.10 \pm 0.02(2)$ \\
\hline BG Lac & $-0.01(4)$ & T Vel & $-0.02(5)$ \\
\hline BN Pup & $+0.01(4)$ & $\mathrm{T}$ Vul & $+0.01 \pm 0.02$ \\
\hline CD Cyg & $+0.11 \pm 0.05$ & U Car & $+0.23(7)$ \\
\hline CV Mon & $-0.03(4)$ & U Nor & $+0.13(7)$ \\
\hline del Cep & $+0.08(4,6)$ & U Sgr & $+0.08 \pm 0.02(1)$ \\
\hline DT Cyg & $+0.11(4)$ & UU Mus & $+0.17(7)$ \\
\hline eta Aql & $+0.08 \pm 0.02(1)$ & U Vul & $+0.11 \pm 0.03$ \\
\hline FF Aql & $+0.02(4)$ & V340 Nor & $+0.00(4)$ \\
\hline FM Aql & +0.08 (4) & V350 Sgr & $+0.18(6)$ \\
\hline FN Aql & $-0.02(4)$ & V496 Aql & +0.05 (4) \\
\hline KN Cen & $+0.23(7)$ & V Car & $+0.02(7,8)$ \\
\hline KQ Sco & $+0.16(4)$ & V Cen & +0.04 (4) \\
\hline $1 \mathrm{Car}$ & $+0.13(7,8)$ & VW Cen & $+0.04(7)$ \\
\hline LS Pup & $-0.10(7)$ & VY Car & $+0.03(8)$ \\
\hline RS Pup & $+0.17(5)$ & VZ Cyg & $+0.05(6)$ \\
\hline RT Aur & $+0.06(4)$ & VZ Pup & $-0.15(4)$ \\
\hline RU Sct & $+0.01(4)$ & W Sgr & $+0.03 \pm 0.03$ \\
\hline RY Sco & +0.09 (6) & WZ Car & $+0.14(7)$ \\
\hline RY Vel & $-0.03(5)$ & WZ Sgr & $+0.20 \pm 0.05$ \\
\hline RZ Vel & $-0.07(5)$ & X Cyg & $+0.10 \pm 0.03(2)$ \\
\hline S Mus & $+0.19(7)$ & X Lac & $-0.02(6)$ \\
\hline S Nor & $+0.05(4)$ & X Pup & $-0.03 \pm 0.03$ \\
\hline S Sge & $+0.10 \pm 0.02$ & X Sgr & $-0.29(4)$ \\
\hline SS Sct & $+0.06(6)$ & XX Cen & $+0.10(7)$ \\
\hline SU Cas & $-0.01(4)$ & Y Lac & $-0.04 \pm 0.06$ \\
\hline SU Cyg & $-0.03 \pm 0.04$ & Y Oph & +0.05 (4) \\
\hline S Vul & $-0.03 \pm 0.03$ & Y Sgr & $+0.05 \pm 0.03$ \\
\hline SV Vul & $+0.05 \pm 0.04$ & YZ Sgr & $+0.08(1)$ \\
\hline SW Vel & $-0.03(4,5)$ & zeta Gem & $+0.04(4)$ \\
\hline SX Vel & $-0.03(5)$ & Z Lac & $+0.01 \pm 0.03(2)$ \\
\hline
\end{tabular}

$1=$ Luck \& Andrievsky (2004); $2=$ Kovtyukh et al. (2005b); $3=$ Andrievsky et al. (2005); $4=$ Andrievsky et al. (2003); $5=$ Luck et al. (2003); 6 = Luck et al. (2006); 7 = Mottini et al. (2008); $8=$ Lemasle et al. (2007).

A weighted fit to the RV curve was performed using MRQMIN with $(2 N+2)+5$ free parameters (sometimes less when e.g. the eccentricity was fixed), with $N$ the number of Fourier terms in the pulsation curve. This procedure does not take into account the possibility that the period also changed over the time spanned by the RV data.

As the data sometimes spans more than a hundred years of data with possible shifts in zero points and varying intrinsic accuracies, the fitting was done as follows. Initially all data points were given the same error in RV, and the fit was made. A mean offset and rms per dataset between data and model was calculated. If the offset was more than half the rms, the offset was applied. A second fit was made. The binary motion was subtracted and the datasets compared as outlined above in order to assign realistic error bars per dataset. The fit to the binary + pulsation RV curve was repeated to arrive at the final fit.

The orbital elements are listed in Table 4. For most of the known spectroscopic binaries the derived orbital parameters are close to literature values, cf. Evans et al. (1990) for FF Aql, Imbert (1996) for Z Lac and U Vul, Evans et al. (1999) for T Mon, Petterson et al. (2004) for S Mus and V350 Sgr, Benedict et al. (2007) for W Sgr, Gorynya et al. (1995) for S Sge, Evans (1988) for SU Cyg. 
Table 4. Derived orbital parameters of binary Cepheids. Quantities without error bar have been fixed.

\begin{tabular}{rccccc}
\hline \hline Name & $K\left(\mathrm{~km} \mathrm{~s}^{-1}\right)$ & $e$ & $\omega(\mathrm{degr})$ & $T_{0}(\mathrm{JD}-2400000)$ & Period $(\mathrm{d})$ \\
\hline FF Aql & $4.91 \pm 0.07$ & $0.027_{-0.016}^{+0.041}$ & $319 \pm 45$ & $45437 \pm 178$ & $1432.4 \pm 1.1$ \\
S Mus & $14.78 \pm 0.10$ & $0.086_{-0.007}^{+0.008}$ & $194 \pm 4$ & $48575 \pm 5$ & $505.15 \pm 0.08$ \\
S Nor & $2.53 \pm 0.35$ & 0 & 0 & $45639 \pm 67$ & $3584 \pm 33$ \\
S Sge & $15.62 \pm 0.06$ & $0.238_{-0.005}^{+0.005}$ & $202.7 \pm 1.1$ & $48010.7 \pm 1.9$ & $675.72 \pm 0.04$ \\
SU Cas & $0.98 \pm 0.08$ & $0^{+0.004}$ & 0 & $50279 \pm 6$ & $406.76 \pm 0.04$ \\
SU Cyg & $29.83 \pm 0.15$ & $0.350_{-0.003}^{+0.02}$ & $223.85 \pm 0.85$ & $43766.2 \pm 1.1$ & $549.24 \pm 0.02$ \\
T Mon & $8.42 \pm 0.19$ & $0.414_{-0.013}^{+0.013}$ & $204 \pm 3$ & $49300 \pm 143$ & $32449 \pm 726$ \\
U Vul & $3.64 \pm 0.44$ & $0.675_{-0.031}^{+0.033}$ & $353 \pm 4$ & $44800 \pm 16$ & $2510 \pm 2.8$ \\
V350 Sgr & $11.21 \pm 0.12$ & $0.405_{-0.014}^{+0.015}$ & $279 \pm 2$ & $47594 \pm 9$ & $1482 \pm 2.4$ \\
V496 Aql & 3.0 & 0 & 0 & $45606 \pm 25$ & $1331 \pm 6.5$ \\
VZ Cyg & $3.46 \pm 0.19$ & 0 & 0 & $45002 \pm 50$ & $2092 \pm 21$ \\
W Sgr & $1.43 \pm 0.06$ & $0.153_{-0.035}^{+0.046}$ & $331 \pm 16$ & $48380 \pm 79$ & $1651 \pm 8.8$ \\
X Sgr & $2.30 \pm 0.27$ & 0 & 0 & $48208 \pm 19$ & $573.6 \pm 0.6$ \\
XX Cen & $4.47 \pm 0.28$ & 0 & 0 & $44861 \pm 8$ & $924.1 \pm 1.1$ \\
Z Lac & $10.44 \pm 0.10$ & $0.025_{-0.008}^{+0.012}$ & $344 \pm 21$ & $46582 \pm 22$ & $382.63 \pm 0.10$ \\
\hline
\end{tabular}

The only exceptions are VZ Cyg and SU Cas. For VZ Cyg, Gorynya et al. (1995) quote an orbital period of 725 days, a velocity amplitude of $6.5 \mathrm{~km} \mathrm{~s}^{-1}$ and an eccentricity of 0.05 , while Rastorgouev et al. (1997) give very different values of $P=1483 \mathrm{~d}, K=15.1 \mathrm{~km} \mathrm{~s}^{-1}, e=0.74$. After pre-whitening the RV curve with the pulsation period. a Fourier analysis using Period04 indicates a period of 2080 days with an amplitude of $3.3 \mathrm{~km} \mathrm{~s}^{-1}$. Using a larger dataset than the previous papers the eccentric orbit could not be confirmed, and Table 4 lists the derived elements for $e=0$, which results in an orbital period of 2092 days. For SU Cas, Gorynya et al. (1996) quote a period of 408 days, velocity amplitude of $3 \mathrm{~km} \mathrm{~s}^{-1}$ and $e=0.43$, while Rastorgouev et al. (1997) give $P=407 \mathrm{~d}, K=5.2 \mathrm{~km} \mathrm{~s}^{-1}$, $e=0.73$. After pre-whitening the RV curve with the pulsation period, a Fourier analysis using Period04 indicates a period of 408 days with an amplitude of $1.2 \mathrm{~km} \mathrm{~s}^{-1}$. Using the larger dataset than the previous works, the eccentric orbit could not be confirmed, and Table 4 lists the derived elements for $e=0$, which results in an orbital period of 407 days and a small velocity amplitude.

For four stars no orbital elements seem to have been published before. For V496 Aql, Szabados (1989) suggested periods of 1200, 1780, 2700, 3600, 5370, or 10750 days. After pre-whitening the RV curve with the pulsation period a Fourier analysis using Period04 indicates periods of 529, 1355, 1825, 4065 days with an amplitude of $2.6-3.4 \mathrm{~km} \mathrm{~s}^{-1}$.

For a fixed velocity amplitude of $3 \mathrm{~km} \mathrm{~s}^{-1}$ and zero eccentricity the period parameter space was searched for the best fit. This was found for a period of 1331 days, closely followed by a fit with a period of 1769 days. Other periods resulted in much poorer fits. Although clearly more RV data are needed to better determine the orbital parameters, the RV curve was corrected using the parameters listed in Table 4.

For X Sgr a period significantly longer than the 507.25 days suggested by Szabados (1990) is derived even though only a few additional RV have become available since. The orbital elements in Table 4 assume zero eccentricity.

For S Nor, Szabados (1989) suggested periods of 3300 or 6500 days. After pre-whitening the RV curve with the pulsation period a Fourier analysis using Period04 indicates a period of 7910 days with an amplitude of $3.2 \mathrm{~km} \mathrm{~s}^{-1}$. Fixing the eccentricity at zero, the best fitting period is found to be near 3600 days. Although more RV data are needed to better determine the orbital parameters, the RV curve was corrected using the parameters listed in Table 4.

For XX Cen, Szabados (1990) suggested a period of 909 days which is largely confirmed. Although clearly more RV data are needed to better determine the orbital parameters, the RV curve was corrected using the parameters listed in Table 4 which have been derived for zero eccentricity.

Finally, for Y Oph the suggestion in Evans \& Lyons (1986) that there is little evidence for orbital motion is confirmed using significantly more data than they had at their disposal. For LS Pup, Szabados (1996) suggested it to be a binary but no new RV data have been published for this star in the last ten years and no correction for binary motion has been attempted. For Y Sgr, Szabados (1989) suggested a binary period of order 10000-12000 days (and longer could not be excluded). Assuming zero excentricity, several oribital periods longer than 10000 days were tried, but no convincing solution could be found. For BF Oph, Szabados (1989) suggested a binary period of $4420 \pm 80$ days. After pre-whitening the RV curve with the pulsation period, a Fourier analysis using Period04 indicates a period of 4085 days with a velocity amplitude near $3 \mathrm{~km} \mathrm{~s}^{-1}$. The least-squares fitting to the RV data then revealed an alternative good fit with a period of $24700 \pm 2900$ days with an amplitude of $2.5 \mathrm{~km} \mathrm{~s}^{-1}$. In the end the RV data of BF Oph was not corrected.

\section{Results}

\subsection{Distances and magnitudes}

Table 5 lists the distances, radii and absolute magnitudes obtained. The table also lists the adopted $E(B-V)$ and error bar, the derived period, and the $p$-factor following $p=1.376-$ $0.064 \log P$ from Nardetto et al. (2007). The distances, radii and errors scale directly with $p$, so the values for $p=1.33$ are not repeated. The error in the period is a few units in the last decimal place. For the derived quantities two error bars are quoted. For the distance and radius the first error bar listed is the error in the fit, and for the absolute magnitudes the error due to the error in distance and $E(B-V)$. The second error quoted is based on a Monte Carlo simulation where new datasets are generated based on the error bar in each individual $V, K$, RV measurement, and analysed taking an $E(B-V)$ value randomly drawn from a Gaussian distribution based on the listed mean value and 
Table 5. Distances, radii and absolute magnitudes from the BW-analysis.

\begin{tabular}{|c|c|c|c|c|c|c|c|}
\hline Name & $\overline{E(B-V)}$ & Period (d) & $\bar{p}$ & $d$ & 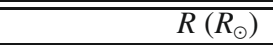 & $\overline{\bar{M}}$ & $M_{K}$ \\
\hline AQ Pup & $0.518 \pm 0.010$ & 30.0980 & 1.281 & $3292.2 \pm 80.0 \pm 125.8$ & $149.7 \pm 3.7 \pm 6.0$ & $-5.51 \pm 0.06 \pm 0.09$ & $-7.44 \pm 0.05 \pm 0.09$ \\
\hline BB Sgr & $0.281 \pm 0.009$ & 6.637111 & 1.323 & $768.7 \pm 25.0 \pm 25.7$ & $46.9 \pm 1.5 \pm 1.5$ & $-3.40 \pm 0.08 \pm 0.08$ & $-5.00 \pm 0.07 \pm 0.07$ \\
\hline BF Oph & $235 \pm 0.010$ & 4.067677 & .337 & $727.7 \pm 32.6 \pm 28.9$ & $31.9 \pm 1.4 \pm 1.3$ & $-2.72 \pm 0.10 \pm 0.09$ & $-4.20 \pm 0.10 \pm 0.09$ \\
\hline BG Lac & $0.300 \pm 0.016$ & 5.33192 & 329 & $1700.9 \pm 24.3 \pm 38.5$ & $39.9 \pm 0.6 \pm 0.9$ & $-3.24 \pm 0.06 \pm 0.08$ & $-4.70 \pm 0.03 \pm 0.05$ \\
\hline BN Pup & $416 \pm 0.018$ & 13.67243 & 303 & $3773.4 \pm 94.6 \pm 145.9$ & $80.2 \pm 2.0 \pm 3.2$ & $-4.30 \pm 0.08 \pm 0.11$ & $-6.08 \pm 0.05 \pm 0.09$ \\
\hline CD Cyg & $493 \pm 0.015$ & 17.07406 & 297 & $.7 \pm 64.1 \pm 60.5$ & $88.3 \pm 2.4 \pm 2.2$ & $-4.46 \pm 0.08 \pm 0.08$ & $-6.31 \pm 0.06 \pm 0.06$ \\
\hline CV Mon & $722 \pm 0.022$ & 5.378662 & 329 & $1475.1 \pm 60.7 \pm 53.9$ & $36.7 \pm 1.5 \pm 1.3$ & $-2.92 \pm 0.11 \pm 0.12$ & $-4.48 \pm 0.09 \pm 0.08$ \\
\hline DT Cyg & $042 \pm 0.011$ & 2.499189 & .351 & $03.3 \pm 75.7 \pm 66.5$ & $34.9 \pm 4.4 \pm 3.9$ & $-3.26 \pm 0.26 \pm 0.29$ & $-4.48 \pm 0.26 \pm 0.28$ \\
\hline eta Aql & $0.130 \pm$ & 7.176813 & 1.321 & $.7 \pm 7.1$ & $49.3 \pm 1.3 \pm 1.3$ & $-3.59 \pm 0.06 \pm 0.07$ & $-5.14 \pm 0.05 \pm 0.06$ \\
\hline FM Aql & $589 \pm$ & 274 & 326 & $1141.2 \pm ?$ & $55.1 \pm 1.9 \pm 1.8$ & $-3.93 \pm 0.08=$ & $-5.40 \pm 0.07 \pm 0.07$ \\
\hline FN Aql & $483 \pm$ & 48163 & 313 & $1217.5 \pm 2$ & $52.2 \pm 1.2 \pm 1.1$ & $-3.62 \pm 0.06 \pm 0.06$ & $-5.21 \pm 0.05=$ \\
\hline KN Cen & $797 \pm$ & 34.0498 & 278 & \pm 9 & $7.1 \pm 4.2 \pm 4.5$ & $-5.71 \pm 0.30 \pm$ & $-7.74 \pm$ \\
\hline KQ Sco & $869 \pm 0.021$ & 28.6958 & 283 & 5.8 & $4 \pm 6.2$ & $-5.13 \pm 0$ & $-7.43 \pm$ \\
\hline LS Pup & $0.461 \pm 0.015$ & 14.14725 & .302 & 157.8 & $85.4 \pm 1.6 \pm 2.7$ & $-4.49 \pm 0$ & $-6.24 \pm($ \\
\hline RS Pup & $0.457 \pm 0.009$ & 41.457 & 272 & \pm 65.2 & \pm 6.8 & $-5.67 \pm$ & $-7.71 \pm$ \\
\hline RU Sct & $0.921 \pm 0.012$ & 19.7032 & 293 & 60.9 & \pm 3.2 & $0.09 \pm 0.08$ & $-6.76 \pm$ \\
\hline RY Sco & $0.718 \pm 0.018$ & 20.32134 & 292 & 56.8 & \pm 4.4 & $-4.68 \pm 0.08 \pm 0.13$ & $-6.44 \pm 0.05$ \\
\hline RY Vel & $0.547 \pm 0.010$ & 28.1240 & 283 & \pm 95.3 & \pm 5.0 & $-5.08 \pm 0.04 \pm 0.10$ & $-6.92 \pm 0.03$ \\
\hline RZ Vel & $0.299 \pm 0.010$ & 20.3996 & 1.292 & \pm 27.1 & $\pm 1.3 \pm 1.8$ & $-4.67 \pm 0.04 \pm 0.06$ & $-6.61 \pm 0.03 \pm 0.04$ \\
\hline S Mus & $0.212 \pm 0.016$ & 997 & 1.313 & 24.2 & \pm 1.8 & $-4.03 \pm 0.10 \pm 0.09$ & 0.07 \\
\hline S Nor & $0.179 \pm$ & 425 & .313 & & & $\pm 0.06 \pm$ & 0.06 \\
\hline S Sge & $0.100 \pm($ & 207 & 317 & & & 06 & -5.3 \\
\hline SS Sct & $0.325 \pm$ & & 0 & & 2.4 & -2 & -4.1 \\
\hline SU Cas & 0.25 & & 7 & 2.9 & .6 & -2.6 & $-3.71 \pm$ \\
\hline SU Cyg & 0.09 & & 39 & .0 & .5 & $-3 .(1+x) \cdot(x)$ & -4.2 \\
\hline S Vul & $0.727 \pm 0$ & 714 & 1.258 & $=205.7$ & \pm 12.7 & -6. & $-8 . \varepsilon$ \\
\hline SV Vul & $0.461 \pm($ & 028 & .270 & 58.2 & $0 \pm 7.8$ & -6 & $-8.15 \pm$ \\
\hline SW Vel & $0.344 \pm$ & 95 & 288 & 4.9 & $2 \pm 2.5$ & -4 & -6.6 \\
\hline SX Vel & $0.236 \pm 0.012$ & 042 & 1.313 & \pm 121.5 & $1 \pm 3.7$ & $-3.74 \pm 0.09 \pm$ & $-5.33 \pm 0.08 \pm 0.15$ \\
\hline SZ Aql & $0.537 \pm 0.017$ & 977 & 297 & \pm 47.3 & \pm 2.3 & $-4.54 \pm 0.06 \pm 0.09$ & $-6.46 \pm 0.03 \pm 0.06$ \\
\hline SZ Tau & $0.295 \pm 0.011$ & 3.148921 & 344 & \pm 21.6 & \pm 1.4 & $-3.05 \pm 0.06 \pm 0.10$ & $-4.38 \pm 0.05 \pm 0.09$ \\
\hline T Mon & $0.181 \pm 0.011$ & .0318 & 1.284 & $.0 \pm 19.6$ & $7 \pm 2.0 \pm 1.8$ & $-4.88 \pm 0.05 \pm 0.06$ & $-6.98 \pm 0.03 \pm 0.04$ \\
\hline TT Aql & $0.438 \pm 0.011$ & 502 & 1.303 & $1025.1 \pm 22.1 \pm 27.2$ & $0 \pm 1.8 \pm 2.1$ & $-4.32 \pm 0$ & $-6.12 \pm 0$ \\
\hline $\mathrm{T}$ Vel & $0.289 \pm($ & 4.639811 & 1.333 & \pm 27.5 & $\pm 0.3 \pm 1.0$ & $-2.78 \pm 0.04 \pm 0.07$ & $-4.31 \pm 0.02 \pm 0.06$ \\
\hline U Car & 0.26 & & .274 & 2.8 & 1.5 & -5 . & 0.06 \\
\hline U Nor & $0.862 \pm$ & 18 & 5 & 2.0 & .6 & -4 & -6 . \\
\hline U Sgr & 0.40 & 06 & 3 & 2.4 & .8 & -3 . & -4.8 \\
\hline UU Mus & 0 . & & & 290 & .5 & -3 . & -5.6 \\
\hline U Vul & $0.603 \pm$ & & 8 & & .7 & -3 & -5.2 \\
\hline 0 Nor & 8 & & 1.309 & .9 & .2 & -3 . & -5 . \\
\hline & $0.299 \pm$ & 48 & 1. & 0 & 6 & -3 . & -4 \\
\hline V496 Aql & $7 \pm$ & 02 & 3 & .7 & 1.0 & -3. & $-4.94 \pm$ \\
\hline V Car & $0.169 \pm 0.011$ & 6.696707 & 1.323 & $908.5 \pm 28.9 \pm 47.0$ & $38.2 \pm 1.2 \pm 2.0$ & $-2.96 \pm 0.08 \pm 0.12$ & $-4.56 \pm 0.07 \pm 0.12$ \\
\hline V Cen & $0.292 \pm 0.012$ & 5.494010 & 1.329 & $672.6 \pm 16.5 \pm 20.7$ & $40.2 \pm 1.0 \pm 1.2$ & $-3.25 \pm 0.07 \pm 0.08$ & $-4.72 \pm 0.05 \pm 0.07$ \\
\hline VW Cen & $0.428 \pm 0.024$ & 15.03733 & 1.301 & $4 \pm 66.8 \pm 120.1$ & $.7 \pm 1.6 \pm 2.7$ & $-3.96 \pm 0.09 \pm 0.12$ & $-6.15 \pm 0.04 \pm 0.07$ \\
\hline VY Car & $0.237 \pm 0.009$ & 9050 & 1.294 & $2 \pm 36.6$ & $5 \pm 1.9 \pm 2.0$ & $-4.11 \pm 0.06 \pm 0.06$ & $-6.07 \pm 0.05 \pm 0.06$ \\
\hline VZ Cyg & $0.266 \pm 0.011$ & 6440 & 1.332 & \pm 54.5 & $4 \pm 1.1 \pm 1.2$ & $-3.13 \pm 0.07 \pm 0.08$ & $-4.57 \pm 0$ \\
\hline VZ Pup & $0.459 \pm 0.011$ & 23.17494 & 1.289 & $4315.0 \pm 86.7 \pm 162.2$ & $7 \pm 2.0 \pm 4.0$ & $-4.98 \pm 0.06 \pm 0.10$ & $-6.63 \pm 0.04 \pm 0.09$ \\
\hline WZ Car & $0.370 \pm$ & 23.01521 & 1.289 & 5.7 & 1.8 & $5 \pm 0.06$ & $-6.59 \pm$ \\
\hline WZ Sgr & $0.431 \pm 0.011$ & 8501 & 1.290 & $1754.1 \pm 29.8 \pm 26.8$ & $117.6 \pm 2.0 \pm 1.7$ & $-4.55 \pm 0.05 \pm 0.06$ & $-6.77 \pm 0.04 \pm 0.04$ \\
\hline X Cyg & $0.228 \pm 0.012$ & .3856 & 1.298 & $1081.0 \pm 25.3 \pm 22.7$ & $92.8 \pm 2.2 \pm 1.9$ & $-4.49 \pm 0.06 \pm 0.07$ & $-6.39 \pm 0.05 \pm 0.05$ \\
\hline X Lac & $0.336 \pm 0.011$ & 5.44453 & 1.329 & $1507.3 \pm 83.0 \pm 70.9$ & $3 \pm 2.4 \pm 2.1$ & $-3.59 \pm 0.12 \pm 0.12$ & $-4.95 \pm 0.12 \pm 0.11$ \\
\hline X Pup & $0.402 \pm 0.009$ & 657 & 1.285 & $2779.4 \pm 48$ & $117.0 \pm 2.0 \pm 5.8$ & $-4.95 \pm 0.05 \pm 0.11$ & $-6.90 \pm$ \\
\hline XX Cen & $0.266 \pm 0.011$ & 10.95351 & 1.309 & $9 \pm 31.9$ & $58.0 \pm 1.4 \pm 1.2$ & $-3.77 \pm 0.06 \pm 0.06$ & $-5.42 \pm 0.05 \pm 0.05$ \\
\hline Y Lac & $0.207 \pm 0.016$ & 4.323760 & 1.335 & $2523.9 \pm 7$ & $41.8 \pm 1.2 \pm 1.4$ & $-3.52 \pm 0.08 \pm 0.10$ & $-4.85 \pm 0.06 \pm 0.07$ \\
\hline Y Oph & $0.645 \pm 0.015$ & 17.12614 & 1.297 & $594.4 \pm 17.5 \pm 21.0$ & $85.8 \pm 2.5 \pm 3.0$ & $-4.84 \pm 0.08 \pm 0.10$ & $-6.33 \pm 0.06 \pm 0.08$ \\
\hline YZ Sgr & $0.281 \pm($ & & 1.313 & $1102.4 \pm 53.9 \pm 90.8$ & $57.2 \pm 2.8 \pm 4.8$ & $-3.77 \pm 0.11 \pm 0.19$ & $-5.41 \pm 0.10 \pm 0.19$ \\
\hline Z Lac & $0.370 \pm 0.011$ & 10.88569 & 1.310 & $1877.5 \pm 37.4 \pm 40.1$ & $68.4 \pm 1.4 \pm 1.5$ & $-4.14 \pm 0.06 \pm 0.07$ & $-5.78 \pm 0.04 \pm 0.05$ \\
\hline
\end{tabular}

$1 \sigma$ error bar. The second error quoted is the $1 \sigma$ dispersion in the derived quantities.

Figure 1 illustrates the fit to the $V, K$, and RV curve for AQ Pup, while Fig. 2 shows the variation of the angular diameter against phase and the change in angular diameter derived from the SB relation against the change in radius from integration of the RV curve from which the distance is derived (see Eq. (5)).
Figures similar to 1 and 2 for all stars in the sample are available from the author.

For ten stars the best available distance comes from the HSTbased parallax (Benedict et al. 2007). Although a BW-type analysis has been carried out for these stars (also see G07), only the mean $V$ and $K$ magnitudes derived here have been used together 

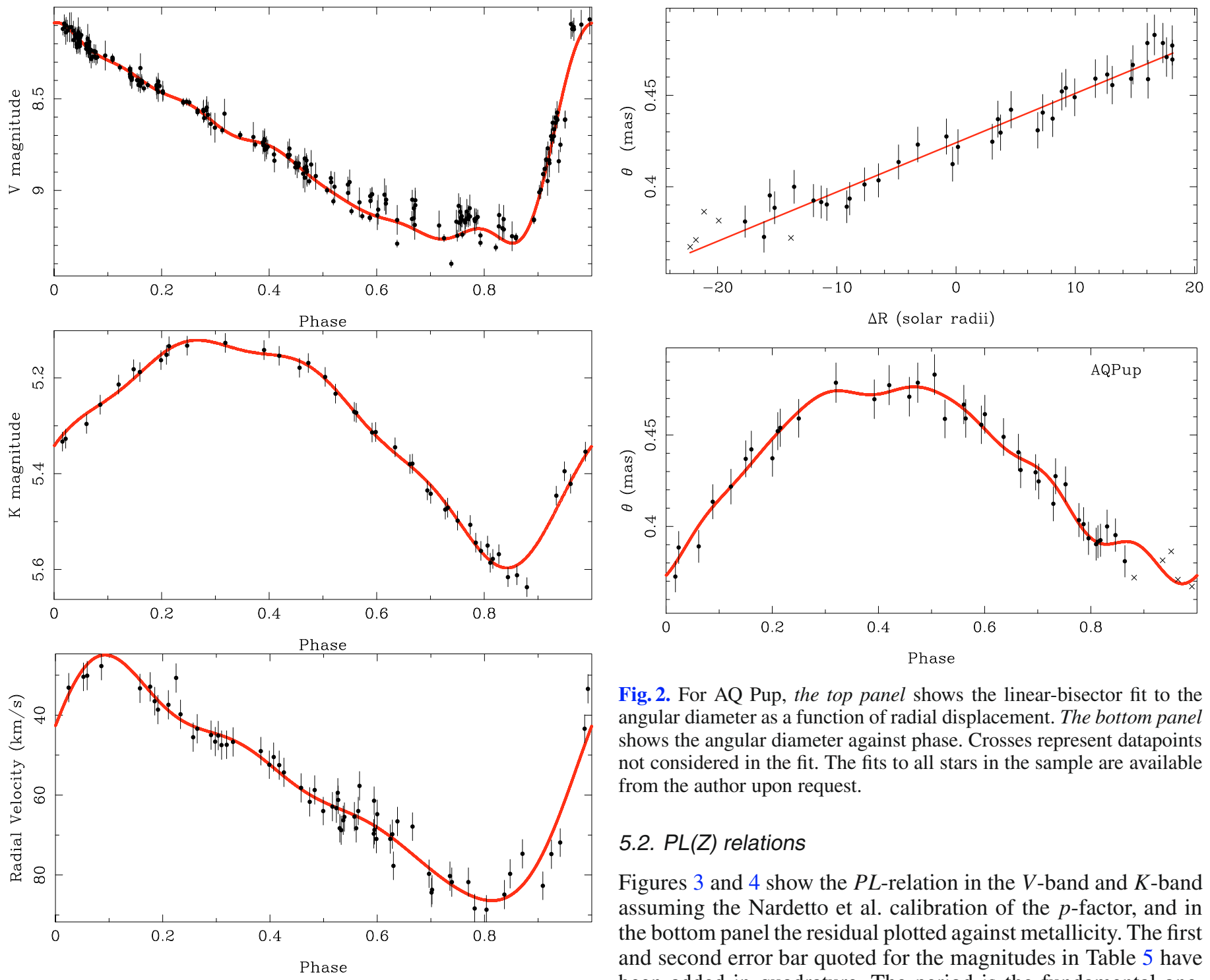

Fig. 1. The phased curves in $V, K$ and RV are shown for AQ Pup. Data points are shown with errors bars and the line shows the harmonic fit. The fits to all stars in the sample are available from the author upon request.

with the parallax from Benedict et al. to derive the absolute magnitudes listed in Table 6.

F07 studied the calibration of the Galactic $P L$-relation in various photometric bands using a set of 59 calibrating stars, with distances from HST and revised Hipparcos parallaxes, interferometric Baade-Wesselink and infrared SB parallaxes and Main-Sequence fitting.

Of the 58 stars in Table 5, 57 have a distance quoted in F07. The ratio of the distance quoted in Table 5 and that in Table 6 of finally adopted parallaxes in F07 is $1.02 \pm 0.11$ (minus one outlier, X Lac). The absolute difference in distance between the two estimates is 1.1 times the combined error bar. Regarding the absolute magnitudes quoted in their Table 7, one difference w.r.t. the values in Table 5 is due to the fact that F07 use published intensity-mean values, while in the present paper these have been derived in a self-consistent way from the Fourier fit to the data.

Fig. 2. For AQ Pup, the top panel shows the linear-bisector fit to the angular diameter as a function of radial displacement. The bottom panel shows the angular diameter against phase. Crosses represent datapoints not considered in the fit. The fits to all stars in the sample are available from the author upon request.

\section{2. $P L(Z)$ relations}

Figures 3 and 4 show the $P L$-relation in the $V$-band and $K$-band assuming the Nardetto et al. calibration of the $p$-factor, and in the bottom panel the residual plotted against metallicity. The first and second error bar quoted for the magnitudes in Table 5 have been added in quadrature. The period is the fundamental one, and the classification into fundamental and first overtone pulsator is taken from F07. Four stars in the sample are overtone pulsators (DT Cyg, SU Cas, SZ Tau, X Lac) and their period is fundamentalised using an iterative procedure using the formula (Alcock et al. 1995)

$P_{1} / P_{0}=0.720-0.027 \log P_{0}$.

The metallicity dependence has been determined in two ways, one by first fitting a linear $P L$-relation, and fitting the residual with a linear relation against $[\mathrm{Fe} / \mathrm{H}]$ (as shown in Figs. 3 and 4), and secondly by making a linear-fit in the two variables $\log P$ and $[\mathrm{Fe} / \mathrm{H}]$, as quoted below. The results are essentially the same.

For the adopted $p$-factor dependence on period the PLZrelation in $V$ becomes

$$
\begin{aligned}
M_{V}= & (-2.60 \pm 0.09) \log P+(-1.30 \pm 0.10) \\
& +(+0.27 \pm 0.30)[\mathrm{Fe} / \mathrm{H}],
\end{aligned}
$$

and in $K$

$$
\begin{aligned}
M_{K}= & (-3.38 \pm 0.08) \log P+(-2.19 \pm 0.09) \\
& +(-0.11 \pm 0.24)[\mathrm{Fe} / \mathrm{H}] .
\end{aligned}
$$

For a constant $p$-factor of 1.33 this would have become

$M_{V}=(-2.72 \pm 0.09) \log P+(-1.23 \pm 0.10)$

$$
+(+0.24 \pm 0.30)[\mathrm{Fe} / \mathrm{H}] \text {, }
$$


Table 6. Absolute magnitudes for the Cepheids with an HST based parallax.

\begin{tabular}{rcccc}
\hline \hline Name & $E(B-V)$ & $\pi$ & $M_{V}$ & $M_{K}$ \\
\hline beta Dor & $0.076 \pm 0.015$ & $3.14 \pm 0.16$ & $-4.01 \pm 0.12$ & $-5.58 \pm 0.11$ \\
del Cep & $0.070 \pm 0.010$ & $3.66 \pm 0.15$ & $-3.43 \pm 0.09$ & $-4.87 \pm 0.09$ \\
FF Aql & $0.194 \pm 0.018$ & $2.18 \pm 0.18$ & $-3.02 \pm 0.15$ & $-4.32 \pm 0.14$ \\
l Car & $0.158 \pm 0.018$ & $2.01 \pm 0.20$ & $-5.25 \pm 0.22$ & $-7.43 \pm 0.21$ \\
RT Aur & $0.061 \pm 0.024$ & $2.40 \pm 0.19$ & $-2.82 \pm 0.18$ & $-4.19 \pm 0.17$ \\
T Vul & $0.103 \pm 0.018$ & $1.90 \pm 0.23$ & $-3.18 \pm 0.26$ & $-4.43 \pm 0.25$ \\
W Sgr & $0.112 \pm 0.009$ & $2.28 \pm 0.20$ & $-3.90 \pm 0.19$ & $-5.43 \pm 0.18$ \\
X Sgr & $0.176 \pm 0.030$ & $3.00 \pm 0.18$ & $-3.62 \pm 0.16$ & $-5.14 \pm 0.13$ \\
Y Sgr & $0.203 \pm 0.012$ & $2.13 \pm 0.29$ & $-3.26 \pm 0.28$ & $-4.80 \pm 0.28$ \\
zeta Gem & $0.018 \pm 0.010$ & $2.78 \pm 0.18$ & $-3.94 \pm 0.14$ & $-5.65 \pm 0.14$ \\
\hline
\end{tabular}
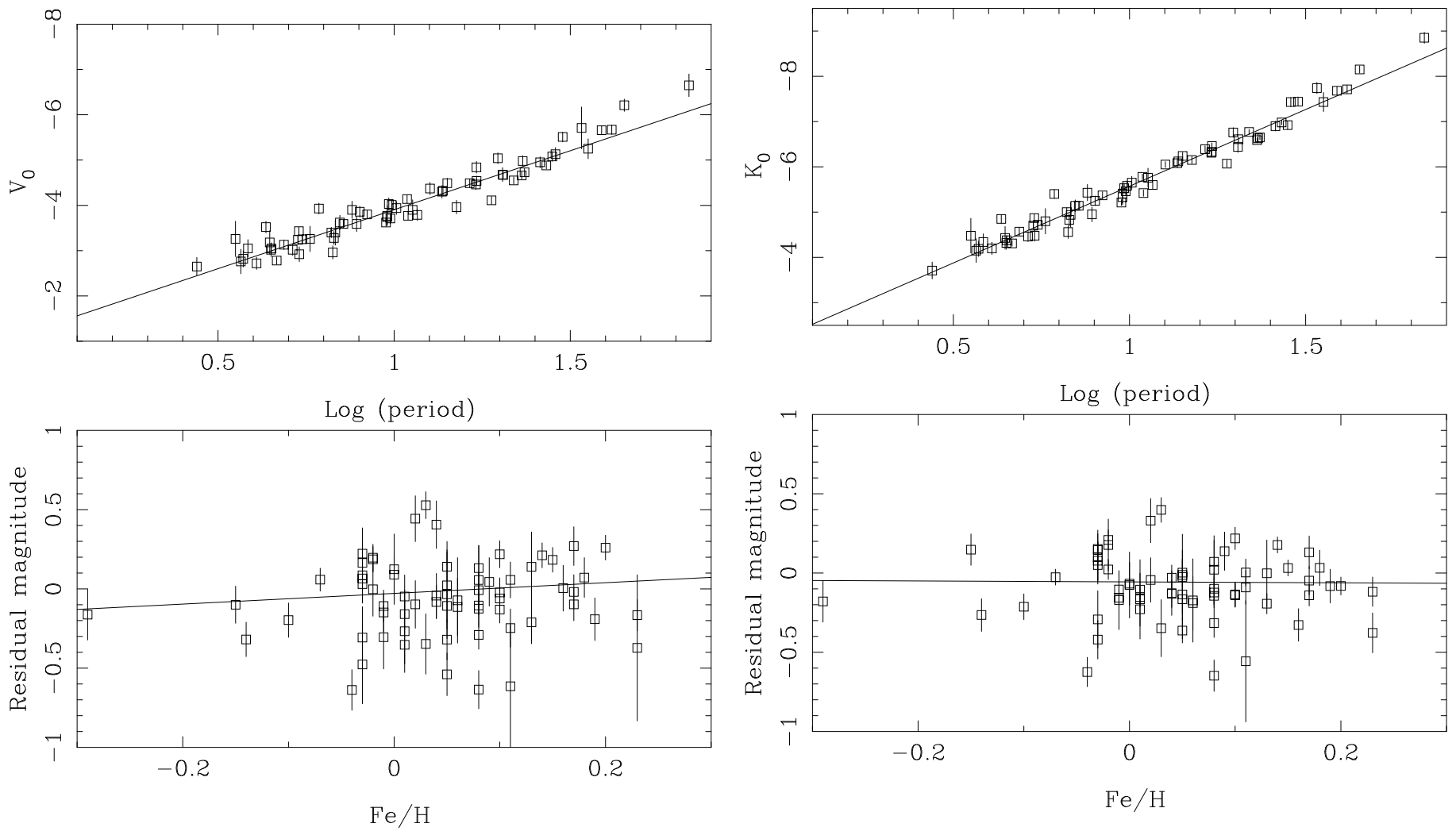

Fig. 3. Period-Luminosity relation in the $V$-band. The bottom panel shows the residual plotted versus metallicity. Lines indicate the fits to the data.

and in $K$

$$
\begin{aligned}
M_{K}= & (-3.50 \pm 0.08) \log P+(-2.09 \pm 0.09) \\
& +(-0.10 \pm 0.24)[\mathrm{Fe} / \mathrm{H}] .
\end{aligned}
$$

Ignoring the metallicity dependence results in $P L$-relations that have slopes and zeropoints that differ in the third decimal from the ones quoted above.

Ignoring RS Pup, SV Vul and S Vul which have the longest periods, and hence limiting the period to $<40$ days results in

$$
\begin{aligned}
M_{V}= & (-2.50 \pm 0.10) \log P+(-1.40 \pm 0.11) \\
& +(+0.14 \pm 0.31)[\mathrm{Fe} / \mathrm{H}],
\end{aligned}
$$

and in $K$

$$
\begin{aligned}
M_{K}= & (-3.29 \pm 0.08) \log P+(-2.28 \pm 0.10) \\
& +(-0.01 \pm 0.26)[\mathrm{Fe} / \mathrm{H}] .
\end{aligned}
$$

Fig. 4. Period-Luminosity relation in the $K$-band. The bottom panel shows the residual plotted versus metallicity. Lines indicate the fits to the data.

For a constant $p$-factor of 1.33 this would have become

$$
\begin{aligned}
M_{V}= & (-2.60 \pm 0.10) \log P+(-1.33 \pm 0.11) \\
& +(+0.11 \pm 0.31)[\mathrm{Fe} / \mathrm{H}],
\end{aligned}
$$

and in $K$

$$
\begin{aligned}
f M_{K}= & (-3.41 \pm 0.08) \log P+(-2.18 \pm 0.10) \\
& +(-0.02 \pm 0.25)[\mathrm{Fe} / \mathrm{H}] .
\end{aligned}
$$

The effect of the negative $\log P$ dependence on $p$ compared to a constant value is to flatten the $P L$-relation. The size of the effect is expected to be about $5 \log (1+0.064 / 1.33) \approx 0.10$ and this is indeed reflected in the fitted slope. This change in slope is about equal to the $1 \sigma$ error bar derived in the slope.

F07 quotes slopes of $-2.77 \pm 0.08$ in $V$ and $-3.37 \pm 0.06$ in $K$ based on 58 stars. For the LMC they combine data for the OGLE Cepheid sample (see below) with data from Persson et al. (2004). They find a slope of $-2.73 \pm 0.03$ in $V$, and $-3.23 \pm 0.03$ in $K$. The conclusion by F07 is that Galactic and LMC slopes are in good agreement. 
There are some issues however that may make a direct comparison between the LMC and the Galaxy less obvious. The distribution of the Galactic Cepheids that define the calibration is more or less uniform in $\log P$ (both in $\mathrm{F} 07$ and in the present study), while the majority of OGLE fundamental mode Cepheids has a $\log P$ between 0.4 and 0.7 and a tail to longer periods (Udalski et al. 1999). In addition it has been demonstrated that the $P L$-relation in the LMC is non-linear in $V$ (e.g. Ngeow et al. 2008, and references therein). Both effects make that the slope based on a least-square fit to the sample of calibrating Galactic Cepheids cannot be compared directly to the slope derived for the LMC sample.

To investigate this further we made use of the data kindly made available by Dr. Soszyński which consists of an OGLE sample of 701 fundamental mode Cepheids whereby the optical light curves have been used to transform the single-epoch 2MASS data into intensity-mean values following the prescription in Soszyński et al. (2005). This is a slightly improved version of the dataset that Dr. Soszyński made available to F07. The mean $V$ magnitude and $E(B-V)$ values are taken from the original OGLE data (Udalski et al. 1999). The 2MASS data were transformed to the SAAO system following Eq. (10) in Koen et al. (2007). Removing outliers with iterative $3 \sigma$ clipping the slope in the LMC $V$-band $P L$-relation becomes $-2.727 \pm 0.033$ based on 664 stars.

In a Monte Carlo simulation a random value of $\log P$ was drawn between 0.45 (the lower limit of the Galactic Cepheids) and 1.6. From the OGLE sample the object closest in $\log P$ was selected and its $V$ and $K$ magnitude taken. In this way samples of 66 stars were generated, and a $P L$-relation fitted. This was repeated a large number of times. The mean slope and the dispersion in the slope is $-2.69 \pm 0.06$.

In the $K$-band, after removing outliers, the $P L$-relation based on 672 stars has a slope of $-3.193 \pm 0.027$. Using the Monte Carlo method with samples of 66 stars the mean slope is $-3.23 \pm 0.05$.

The conclusion is that, within the errors, the distribution of period and/or a non-linearity effect does not bias the derived slope in $V$ and $K$.

In $V$ the observed slope in the LMC agrees within $1 \sigma$ with the one derived for the Galactic sample both for a constant $p$-factor and for the one with a mild period dependence. In $K$ the agreement is less good, in the sense that the $p$-factor with a dependence on period agrees more closely.

\section{Summary and discussion}

The period-luminosity in the $V$ and $K$-band and the dependence on metallicity are investigated for a sample of 68 Galactic Cepheids with an individual metallicity determination from high-resolution spectroscopy. Distances are derived using the Baade-Wesselink technique using the most recent surfacebrightness relation and estimates for the projection factor.

The difference in slope between a constant $p$-factor (consistent with available interferometric data) and a mild dependence on period (following theoretical work by Nardetto et al. 2007) results in a difference in slope in the $P L$-relation which is of the same magnitude as the error in slope.

The slope of the LMC $P L$-relation in $V$ and $K$ is derived taking into account the difference in period distribution between the Galactic sample and the LMC sample. In $V$ the slope for the LMC sample agrees with the galactic one for both assumptions regarding the dependence of $p$ on period. In $K$ the comparison of the slopes favours the mild period dependence of the $p$-factor on period.

The metallicity dependence of the $P L$-relation is investigated and no significant dependence is found. A firm result is not possible as the range in metallicity spanned by the current sample of galactic Cepheids is 0.3-0.4 dex, while previous work suggested a small dependence on metallicity only (typically $-0.2 \mathrm{mag} / \mathrm{dex}$, see the introduction).

It would be extremely valuable to analyse a sample of LMC and SMC Cepheids in a similar way. Unfortunately, the sample of Cepheids with adequate optical, NIR and RV light curves (see e.g. Storm et al. 2004, 2005) for which a Baade-Wesselink distance has been derived (Gieren et al. 2005) and the sample of Magellanic Cloud Cepheids for which a metallicity has been derived from high-resolution spectroscopy (Mottini et al. 2008) have no overlap. High-resolution spectroscopy of MC Cepheids with Baade-Wesselink distances would therefore be extremely valuable.

Acknowledgements. I would like to thank Dr. Igor Soszyński for communicating the intensity-mean 2MASS magnitudes for the OGLE Cepheids, and Maryline Briquet and Djazia Ladjal for taking the Coralie data. The referee pointed out additional metallicity determinations in the literature which led to a significantly larger sample of stars under study. I also thank the ESO librarians Uta Grothkopf and Chris Erdmann for obtaining some of the century old papers with radial velocity data. Part of this research was done while M.G. was a short-term visitor at the Max-Planck Institut für Astrophysik (MPA) in Garching, Germany. This research has made use of the SIMBAD database, operated at CDS, Strasbourg, France.

\section{References}

Abt, H. A. 1959, AJ, 130, 1021

Abt, H. A. 1973, ApJS, 26, 365

Abt, H. A., \& Levy, S. G. 1978, PASP, 90, 188

Adams, W. S., \& Shapley, H. 1918, ApJ, 47, 46

Albrow, M. D., \& Cottrell, P. L. 1996, MNRAS, 280, 917

Alcock, C., Allsman, R. A., Axelrod, T. S., Bennett, D. P., \& Cook, K. H. 1995, AJ, 109, 1653

Andrievsky, S. M., Kovtyukh, V. V., Luck, R. E., et al. 2002a, A\&A, 381, 32

Andrievsky, S. M., Bersier, D., Kovtyukh, V. V., et al. 2002b, A\&A, 384, 140

Andrievsky, S. M., Kovtyukh, V. V., Luck, R. E., et al. 2002c, A\&A, 392, 491

Andrievsky, S. M., Egorova, I. A., Korotin, S. A., \& Kovtyukh, V. V. 2003, AN, 324,532

Andrievsky, S. M., Luck, R. E., Martin P., \& Lépine, J. R. D. 2004, A\&A, 413, 159

Andrievsky, S. M., Luck, R. E., \& Kovtyukh, V. V. 2005, AJ, 130, 1880

Babel, J., Burki, G., Mayor, M., Chmielewski, Y., \& Waelkens, C. 1989, A\&A, 216, 125

Barnes, T. G., Moffett, T. J., \& Slovak, M. H. 1987, ApJS, 65, 307

Barnes, T. G., Moffett, T. J., \& Slovak, M. H. 1988, ApJS, 66, 43

Barnes, T. G., Fernley, J. A., Frueh, M. L., et al. 1997, PASP, 109, 645

Barnes, T. G., Jeffery, E. J., Berger, J. O., et al. 2003, ApJ, 592, 539

Barnes, T. G., Jeffery, E. J., Montemayor, \& T. J., Skillen, I. 2005, ApJS, 156, 227

Barnes, T. G., Storm, J., Jefferys, W. J., Gieren, W. P., \& Fouqué, P. 2005, ApJ, 631,572

Beavers, W. I. \& Eitter, J. J. 1986, ApJS, 62, 147

Benedict, G. F., McArthur, B. E., Feast, M. W., et al. 2007, AJ, 133, 1810

Berdnikov, L. N., Dambis, A. K., \& Vozyakovs, O. V. 2000, A\&AS, 143, 211

Bersier, D. 2002, ApJS, 140, 465

Bersier, D., Burki, G., Mayor, M., \& Duquennoy, A. 1994, A\&AS, 108, 25

Böhm-Vitense, E., Clark, M., Cottrell, P. L., \& Wallerstein, G. 1990, AJ, 99, 353

Breger, M. 1967, MNRAS, 136, 61

Breger, M. 1970, AJ, 75, 239

Breitfellner, M. G., \& Gillet, D. 1993, A\&A, 277, 541

Caldwell, J. A. R., Coulson, I. M., Dean, J. F., \& Berdnikov, L. N. 2001, JAR 7, 4

Campbell, W. W., \& Moore, J. H. 1928, Lick Obs. Bull., 16, 267

Carter, B. S. 1990, MNRAS, 242, 1

Coulson, I. M. 1983, MNRAS, 203, 925

Coulson, I. M., \& Caldwell, J. A. R. 1985a, SAAO Circ., 9, 5 
Coulson, I. M., \& Caldwell, J. A. R. 1985b, MNRAS, 216, 671

Coulson, I. M., Caldwell, J. A. R., \& Gieren, W. P. 1985, ApJS, 57, 595

Dean, J. F. 1977, MNSSA, 36, 3

Dean, J. F. 1981, SAAOC, 6, 10

Duncan, J. C. 1908, Lick Obs. Bull., 5, 82

Duncan, J. C. 1922, ApJ, 56, 340

Duncan, J. C. 1932, PASP, 44, 324

Eggen, O. J. 1983, AJ, 88, 379

Evans, N. R. 1976, ApJS, 32, 399

Evans, N. R. 1988, ApJS, 66, 343

Evans, N. R., \& Lyons, R. 1986, AJ, 92, 436

Evans, N. R., \& Welch, D. L. 1993, PASP, 105, 836

Evans, N. R., \& Lyons, R. 1994, AJ, 107, 2164

Evans, N. R., \& Sugars, B. J. A. 1997, AJ, 113, 792

Evans, N. R., Welch, D. L., Scarfe, C. D., \& Teays, T. J. 1990, AJ, 99, 1598

Evans, N. R., Welch, D. L., Slovak, M. H., Barnes, T. G. III, \& Moffett, T. J. 1993, AJ, 106, 1599

Evans, N. R., Carpenter, K., Robinson, R., et al. 1999, ApJ, 524, 379

Feast, M. W. 1967, MNRAS, 136, 141

Feast, M. W. 1999, PASP, 111, 775

Feast, M. W., Laney, C. D., Kinman, T. D., van Leeuwen, F., \& Whitelock, P. A. 2008, MNRAS, 386, 2115

Fernley, J. A., Skillen I., \& Jameson, R. F. 1989, MNRAS, 237, 947

Fouqué, P., \& Gieren, W. P. 1997, A\&A, 320, 799

Fouqué, P., Storm, J., \& Gieren, W. P. 2003, in Stellar candles for the extragalactic distance scale, Lect. Notes Phys., 635, 21

Fouqué, P., Arriagada, P., Storm, J., et al. 2007, A\&A, 476, 73 (F07)

Frost, E. B. 1906, ApJ, 23, 264

Fry, A. M., \& Carney, B. W., 1997, AJ, 113, 1073

Gieren, W. P. 1976, A\&A, 47, 211

Gieren, W. P. 1981a, ApJS, 46, 287

Gieren, W. P. 1981b, ApJS, 47, 315

Gieren, W. P. 1985, ApJ, 295, 507

Gieren, W. P. 1989a, A\&A, 216, 135

Gieren, W. P. 1989b, PASP, 101, 160

Gieren, W. P., Barnes T. G., \& Moffett, T. J. 1993, ApJ, 418, 135

Gieren, W. P., Fouqué, P., \& Gómez, M. 1997, ApJ, 488, 74

Gieren, W. P., Fouqué, P., \& Gómez, M. 1998, ApJ, 496, 17

Gieren, W. P., Storm, J., Barnes, T. G., et al. 2005, ApJ, 627, 224

Glass, I. S. 1985, IrAJ, 17, 1

Gorynya, N. A., Samus, N. N., Berdnikov, L. N., Rastorgouev, A. S., \& Sachkov, M. E. 1995, IBVS, 4199

Gorynya, N. A., Samus', N. N., Sachkov, M. E., et al. 1998, PAZh, 24, 939 (VizieR On-line Data Catalog: III/229)

Gould, A. 1994, ApJ, 426, 542

Grayzeck, E. J. 1978, AJ, 83, 1397

Groenewegen, M. A. T. 2007, A\&A, 474, 975

Groenewegen, M. A. T., Romaniello, M., Primas, F., \& Mottini, M. 2004, A\&A, 420,655

Harper, W. E. 1934, Publ. DAO, 6, 151

Häupl, W. 1988, AN, 309, 3

Henden, A. A. 1980, MNRAS, 192, 621

Herbig, G. H., \& Moore, J. H. 1952, ApJ, 116, 348

Imbert, M. 1985, A\&AS, 58, 529

Imbert, M. 1996, A\&AS, 116, 497

Imbert, M. 1999, A\&AS, 140, 79

Isobe, T., Feigelson, E. D., Akritas, M. G., \& Babu, G. J. 1990, ApJ, 364, 104

Jacobsen, T. S. 1970, ApJ, 159, 569

Jacobsen, T. S. 1974, ApJ, 191, 691

Jacobsen, T. S., \& Wallerstein, G. 1981, PASP, 93, 481

Joy, H. A. 1937, ApJ, 86, 363

Joy, H. A. 1952, ApJ, 115, 25

Kennicutt, R. C., Stetson, P. B., Saha, A., et al. 1998, ApJ, 498, 181

Kervella, P., Bersier, D., Mourard D., et al. 2004, A\&A, 428, 587

Kimeswenger, S., Lederle, C., Richichi, A., et al. 2004, A\&A, 413, 1037

Kiss, L. L. 1998, MNRAS, 297, 825

Kiss, L. L. 2000, MNRAS, 314, 420

Kochanek, C. S. 1997, ApJ, 491, 13

Koen, C., Marang, F., Kilkenny, D., \& Jacobs, C. 2007, MNRAS, 380, 1433

Kovtyukh, V. V., Wallerstein, G., \& Andrievsky, S. M. 2005a, PASP, 117, 1173

Kovtyukh V. V., Andrievsky, S. M, Belick, S. I., \& Luck, R. E. 2005b, AJ, 129 , 433

Laney, C. D., \& Caldwell, J. A. R. 2007, MNRAS, 377, 147

Laney, C. D., \& Stobie, R. S. 1992, A\&AS, 93, 93

Lemasle, B., Francois, P., Bono, G., et al. 2007, A\&A, 467, 283

Lenz, P., \& Breger, M. 2005, CoAst, 146, 53

Lloyd Evans, T. 1968, MNRAS, 141, 109

Lloyd Evans, T. 1980, SAAO Circ., 1, 163
Lloyd Evans, T. 1980, SAAO Circ., 1, 257

Luck, R. E., \& Andrievsky, S. M. 2004, AJ, 128, 343

Luck, R. E., Gieren, W. P., Andrievsky, S. M., et al. 2003, A\&A, 401, 939

Luck, R. E., Kovtyukh V. V., \& Andrievsky, S. M. 2006, AJ, 132, 902

Macri, L. M., Stanek, K. Z., Bersier, D., Greenhill, L. J., \& Reid, M. J. 2006, ApJ, 652, 1113

Maddrill, J. D. 1906, PASP, 18, 252

Madore, B. F. 1975, ApJS, 29, 219

Mérand, A., Kervella, P., Coudé du Foresto, V., et al. 2005, A\&A, 438, L9

Mermilliod, J.-C., Mayor, M., \& Burki, G. 1987, A\&AS, 70, 389

Metzger, M. R., Caldwell, J. A. R., \& Schechter, P. L. 1992, AJ, 103, 529

Metzger, M. R., Caldwell, J. A. R., McCarthy, J. K., \& Schechter, P. L. 1993, ApJS, 76, 803

Moffett, T. J., \& Barnes, T. G. 1984, ApJS, 55, 389

Moore, J. H. 1909, Lick Obs. Bull., 5, 111

Mottini, M., Romaniello, M., Primas, F., et al. 2008, A\&A, submitted

Nardetto, N., Fokin, A., Mourard, D., et al. 2004, A\&A, 428, 131

Nardetto, N., Mourard, D., Kervella, P., et al. 2006, A\&A, 453, 309

Nardetto, N., Mourard, D., Mathias, Ph., Fokin, A., \& Gillet, D. 2007, A\&A, 471,661

Ngeow, C., Kanbur, S. M., \& Nanthakumar, A. 2008, A\&A, 477, 621

Niva, G. D., \& Schmidt, E. G. 1979, ApJ, 234, 245

Pel, J. W. 1976, A\&AS, 24, 413

Persson, S. E., Madore, B. F., Krzeminski, W, et al. 2004, AJ, 128, 2239

Petterson, O. K. L., Cottrell, P. L., \& Albrow, M. D. 2004, MNRAS, 350, 95

Petterson, O. K. L., Cottrell, P. L., Albrow, M. D., \& Fokin, A. 2005, MNRAS, 362,1167

Pont, F., Burki, G., \& Mayor, M. 1994, A\&AS, 105, 165

Press, W. H., Teukolsky, S. A., Vetterling, W. T., \& Flannery, B. P. 1992, in Numerical Recipes in Fortran, 77 (Cambridge U.P.)

Rastorgouev, A. S., Gorynya, N. A., \& Samus, N. N. 1997, in Binary Stellar Systems, ed. A. G. Massevich (Moscow: Kozmosinform), 123

Sanford, R. F. 1927, ApJ, 66, 170

Sanford, R. F. 1935, ApJ, 81, 140

Sanford, R. F. 1951, ApJ, 114, 331

Sanford, R. F. 1956, ApJ, 123, 201

Sasselov, D. D., Beauliau, J. P., Renault, C., et al. 1997, A\&A, 324, 471

Schechter, P. L., Avruch, I. M., Caldwell, J. A. R., \& Keane, M. J. 1992, AJ, 104, 1930

Schwarz, G. 1978, Ann. Stat., 6, 461

Shobbrook, R. R. 1992, MNRAS, 255, 486

Slipher, V. M. 1904, ApJ, 20, 146

Soszyński, I., Gieren, W., Pietrzyński, G., et al. 2005, PASP, 117, 823

Stibbs, D. W. N. 1955, MNRAS, 115, 363

Storm, J., Carney, B. W., Gieren, W. P., et al. 2004, A\&A, 415, 531

Storm, J., Gieren, W. P., Fouqué, Barnes, T. G., \& Gómez, M. 2005, A\&A, 440, 487

Sugars, B. J. A., \& Evans, N. R. 1996, AJ, 112, 1670

Szabados, L. 1977, Mitt. Sternw. Ung. Akad. Wiss., Budapest, No. 70

Szabados, L. 1980, Commun. Konkoly Obs. Hung. Acad. Sci., Budapest, No. 76

Szabados, L. 1981, Commun. Konkoly Obs. Hung. Acad. Sci., Budapest, No. 77

Szabados, L. 1989, CoKon, 94, 1

Szabados, L. 1990, MNRAS, 242, 285

Szabados, L. 1991, Commun. Konkoly Obs. Hung. Acad. Sci., Budapest, No. 96

Szabados, L. 1996, A\&A, 311, 189

Szabados, L. 2003, Inf. Bull. Var. Stars, No. 5394

Taylor, M. M., \& Booth, A. J. 1998, MNRAS, 298, 594

Taylor, M. M., Albrow, M. D., Booth, A. J., \& Cottrell, P. L. 1997, MNRAS, 292, 662

ten Bruggencate, P. 1930, Harvard Circ., 351, 1

Turner, D. G., Bryukhanov, I. S., Balyuk, I. I., et al. [arXiv: astro-ph/0709.3085]

Udalski, A., Soszyński, I., Szymański, M., et al. 1999, AcA, 49, 223

van Belle, G. T., Lane, B. F., \& Thompson, R. R. 1999, AJ, 117, 521

van Leeuwen, F. 2007, Hipparcos, the new reduction of the Raw Data, ASSL, 350 (Springer)

van Leeuwen, F., Feast, M. W., Whitelock, P. A., \& Laney, C. D. 2007, MNRAS, 379,23

Wallerstein, G. 1972, PASP, 84, 656

Walraven, J. H., Tinbergen, J., \& Walraven, T. 1964, BAN, 17, 520

Welch, D. L. 1985, Ph.D. thesis, University of Toronto

Welch, D. L., Wieland, F., McAlary, C. W., et al. 1984, ApJS, 54, 547

Wilson, T. D., Carter, M. W., Barnes, T. G., Van Citters, G. W., \& Moffett, T. J. 1989, ApJS, 69, 951

Wisniewski, W. Z., \& Johnson, H. R. 1968, CoLPL, 7, 57

Yong, D., Carney, B. W., Teixera de Almeida, M.-L., \& Pohl, B. L. 2006, AJ, 131,2256 